\title{
Prosthetic Rehabilitation of Midfacial Defect following Cancer Surgery using Three Piece Prosthesis
}

\author{
${ }^{1}$ Sunil Jacob, ${ }^{2}$ Haifa Ashraf \\ ${ }^{1}$ Assistant Professor, Department of Prosthodontics, Sri Ramakrishna Dental College and Hospital, Coimbatore, Tamil Nadu, India \\ ${ }^{2}$ Assistant Professor, Department of Prosthodontics, Riyadh College of Dentistry and Pharmacy, Saudi Arabia
}

Correspondence: Haifa Ashraf, Assistant Professor, Department of Prosthodontics, Riyadh College of Dentistry and Pharmacy Saudi Arabia, e-mail: aifa_abs@yahoo.in

\section{ABSTRACT}

Acquired facial defects may cause functional and psychological impairments that adversely affect a patient's quality of life. Restoration of facial defects can be accomplished surgically, prosthetically or by using a combination of both the methods. The choice depends on many factors, like size, location of the defect and the age of the patient. Reduced vascularity, increased fibrosis and scarring of tissues bordering the defect increase the risk of complications associated with reconstruction. Maxillofacial prostheses have the advantage of not only improving the patient's appearance but also enabling early rehabilitation. This unusual case report details an attempt to rehabilitate a patient who has undergone total maxillectomy and orbital exenteration with the aid of intraoral obturator prosthesis and an extraoral orbital and cheek prosthesis. Keywords: Large facial defect, Total maxillectomy, Orbital exenteration, Orbital prosthesis, Obturator, Cheek prosthesis.

\section{INTRODUCTION}

The fabrication of an extraoral facial prosthesis is as much as an art as it is a science. ${ }^{1}$ Large facial defects involving the oral cavity can be difficult to restore prosthetically because of lack of anatomic undercuts, limited means of retention, mobility of soft tissue margins and the weight of the prosthesis. The challenge of restoring such large facial defects has always perplexed the maxillofacial prosthodontist. ${ }^{2}$ Prosthesis form, coloration and the texture must be as indiscernible as possible from the surrounding natural tissues. The advent of silicone rubber has brought us a material that most nearly meets the requirements of the ideal prosthetic material as outlined by Bulbulian. ${ }^{3}$ Rehabilitation effort can only be successful when patients can appear in public without fear of attracting unwanted attention. $^{4}$

Large facial deformities can result from treatment for tumors, trauma, burns and congenital anomalies. The invasion of cancerous lesions can extend to facial structures adjacent to the tumor site, such as nasal bone, orbital walls or in combination. In such situations, surgical recession results in large deformities which require extensive rehabilitation of both intraoral and extraoral structures. ${ }^{5}$ The use of obturator prosthesis can result in an improvement in speech, mastication, and swallowing by re-establishing the oronasal separation. ${ }^{6}$ Facial prostheses retained with the help of an adhesive, spectacle frames or implants can improve the appearance and the quality of life of the patient. This case report describes the fabrication of both intraoral obturator prosthesis and an extraoral orbital and cheek prosthesis in rehabilitating a patient with a facial defect caused due to total maxillectomy and orbital exenteration of the right side.

\section{CASE REPORT}

A 45-year-old female with poor socioeconomic status was referred by an oral and maxillofacial surgeon to the Department of Prosthodontics at AB Shetty Memorial Institute of Dental Sciences for the prosthetic rehabilitation of a facial defect following surgical recession. The patient was operated following a carcinomatous lesion in the right antrum extending to the infraorbital margin. Clinical features added with radiographic and pathological reports confirmed the presence of squamous cell carcinoma of the right maxillary antrum extending to the floor of the orbit. Total maxillectomy along the midline and orbital exenteration was carried out 7 months back.

\section{Extraoral Examination}

Facial asymmetry present where in the facial prominence of right cheek was lost due to loss of bone support. Scar found in the philtrum region. Sinus opening found just beneath the right eye region (Fig. 1). Absence of right eye with a depressed socket.

\section{Intraoral Examination}

Generalized inflammation with recession of the marginal gingiva present. Palatal mucosa was firm and resilient. Presence of a taught band of tissue on right cheek mucosa due to scarring. 


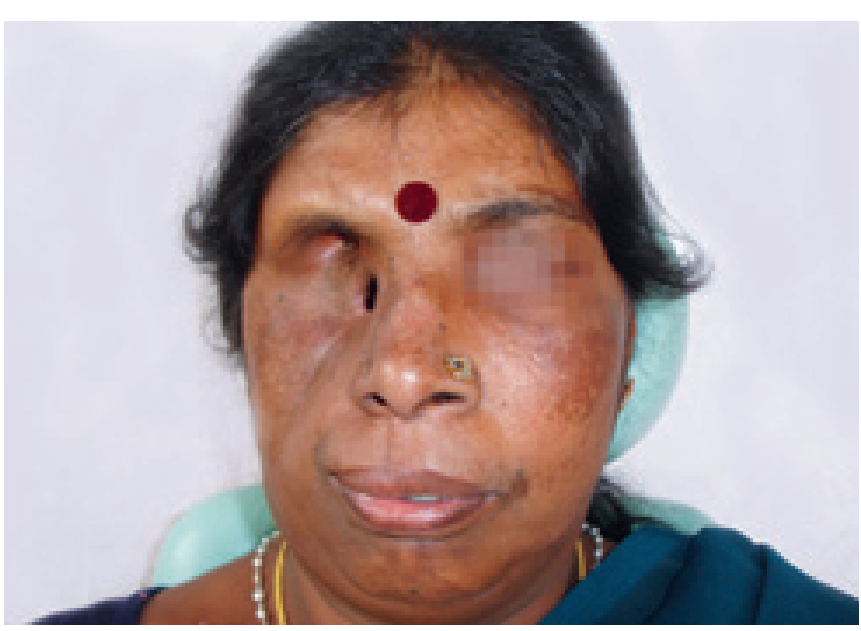

Fig. 1: Patient with right total maxillectomy and orbital defect

Right maxillary segment was missing with an opening into the nasal cavity (Fig. 2). Mandibular arch was partially dentate with missing 37 and 47.

\section{Treatment Plan}

1. Oral prophylaxis and oral hygiene instructions were given. Proper tooth-brushing and flossing was re-emphasized and called for re-evaluation.

2. A three-piece prosthesis was designed to be retained with magnets and a pair of spectacles.

a. Closed hollow bulb obturator prosthesis with cast partial framework design for Aramani class I defect

b. Orbital prosthesis retained with magnets attached to obturator.

c. Cheek prosthesis retained with a pair of spectacles and magnetic attachments to the orbital prosthesis.

\section{Treatment Procedure}

1. Maxillary and mandibular diagnostic impressions were made in irreversible hydrocolloid impression material (Tropicalgin, Zhermack, Italy). Surveying of the maxillary cast was done to design the cast partial denture framework. Design for Aramani Class I maxillectomy defect ${ }^{1}$ was

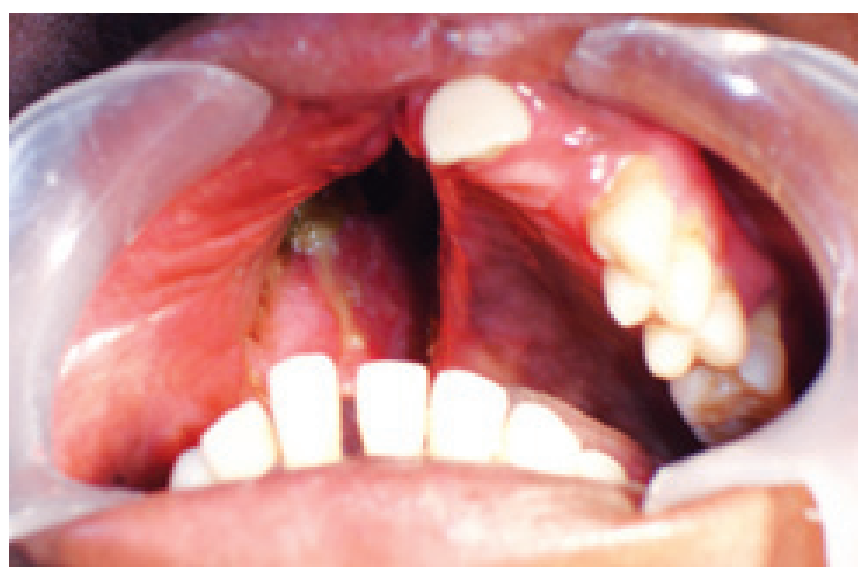

Fig. 2: Maxillary defect area planned and mouth preparation was completed for rest seats and guiding planes. Direct retention was obtained with Akers clasp on premolars and molars and from central incisor with an I-bar clasp. Maxillary arch final impression was made in hydrocolloid impression material (Neocolloid, Zhermack, Italy) poured in die stone (Kalrock; Kalabhai Pvt Ltd, Mumbai, India). Once the surveying, designing and wax block-out were accomplished, refractory cast was poured on to which pattern wax was adapted and a cast partial denture metal framework was casted.

After finishing and polishing, the fit of the framework was checked in the mouth. Molding of the defective area was done with putty elastomeric impression material (Aquasil, Dentsply, Germany) onto the cast framework, care was taken to give adequate support laterally to recompense the collapsed cheek prominence. Final impression of the maxillectomy defect area was made with light body polyvinyl siloxane impression material (Aquasil, Dentsply, Germany) and a pick-up impression was made with irreversible hydrocolloid in a stock tray. Obturator part of the prosthesis was processed and finished in heatpolymerized acrylic resin to make it hollow (Fig. 3). Fit and extension of the obturator part of the prosthesis was checked and verified. Face bow (Artex Quickmount Facebow) transfer done and jaw relations recorded. Teeth arrangement was completed to maximum intercuspation and try-in of the waxed up prosthesis was done in the mouth for esthetics, function and occlusion. Finally, the prosthesis was processed and finished in heat-polymerized acrylic resin. Fit in of the definitive prosthesis was done (Fig. 4).

2. The definitive obturator was then placed in the mouth prior to impression making of the facial defect area. Facial moulage was made using irreversible hydrocolloid material reinforced with gauze and dental plaster. The impression was boxed and poured in Type-III dental stone (Kalastone; Kalabhai Pvt Ltd, Mumbai, India).

3. The wax pattern of the orbit was carved out on the master cast. Care was taken to retain the same characteristics of the contralateral side of the face. An extension was made on the rear surface of the wax model, which extended

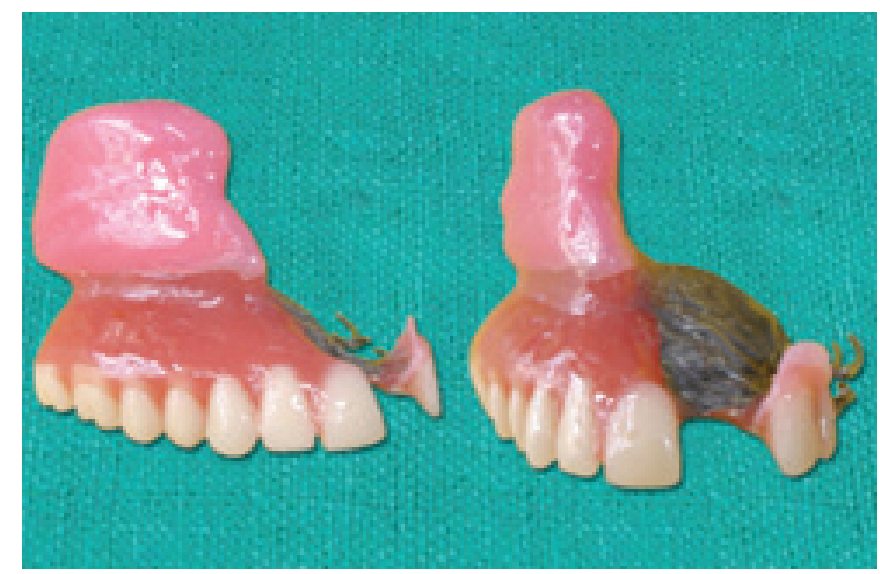

Fig. 3: Final obturator prosthesis 


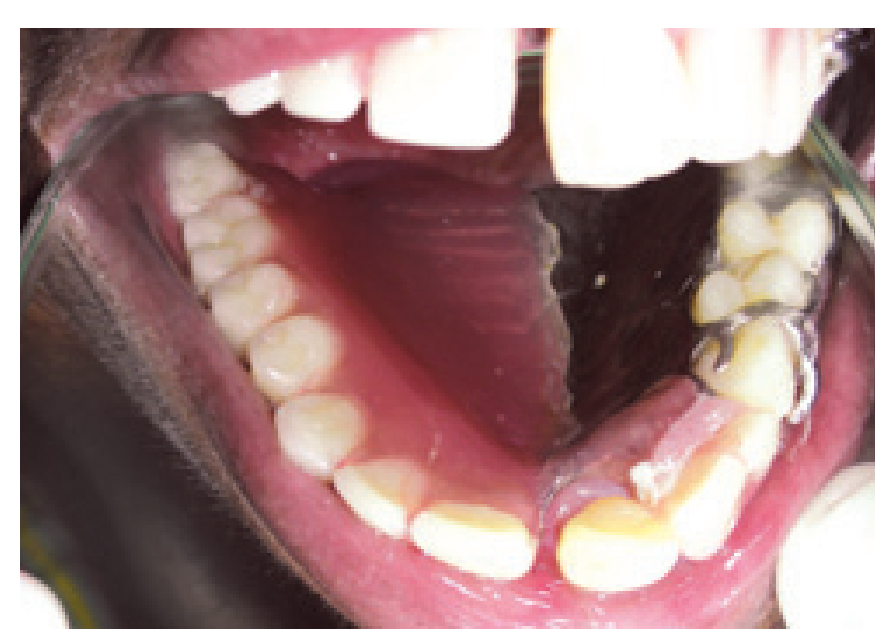

Fig. 4: Obturator fit-in and insertion

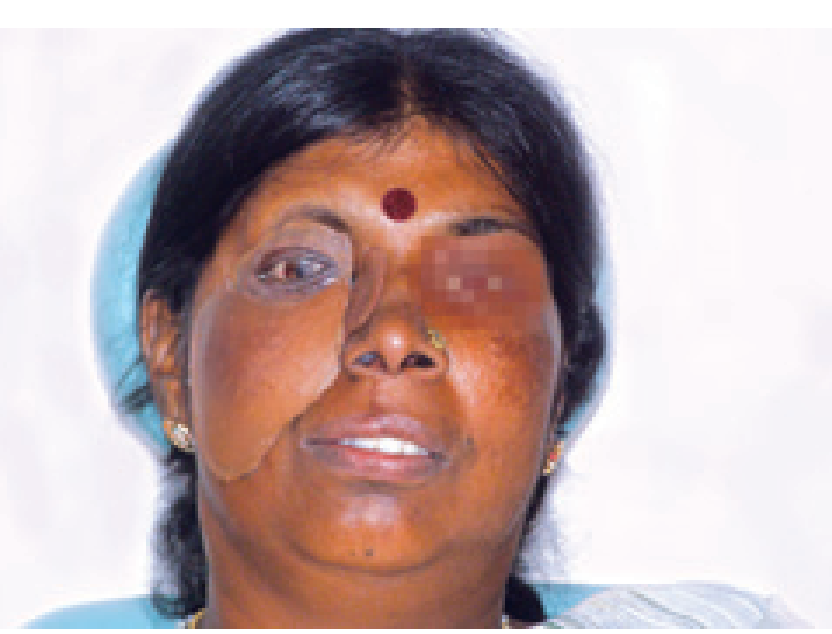

Fig. 6: Definitive prosthesis without spectacles

7. At the time of insertion, the magnets were positioned with auto polymerizing acrylic resin. The cheek prosthesis was then attached to a pair of spectacles using auto polymerizing acrylic resin (Fig. 7).

\section{DISCUSSION}

The problems created by maxillectomy defects are manifested in the functions of chewing, speech and swallowing. Moreover, the patient usually develops esthetic and psychological problems. ${ }^{7}$ Obturator prosthesis is used to restore masticatory function, speech, deglutition and appearance. The design of the metal framework for maxillary obturator varies greatly with different designs. However, the design objective is to select the most suitable components to resist the various forces acting on the obturator prosthesis without applying undue stress on the remaining teeth and soft tissue structures. In an Aramani's class I maxillectomy resection, the dentition and the alveolar bone are removed along the midline. ${ }^{7}$ The design can be either linear or tripodal.

Recording the remaining tissues with least distortion always remains a challenge to the prosthodontist. Difficulty was encountered in removing the impression with extension into

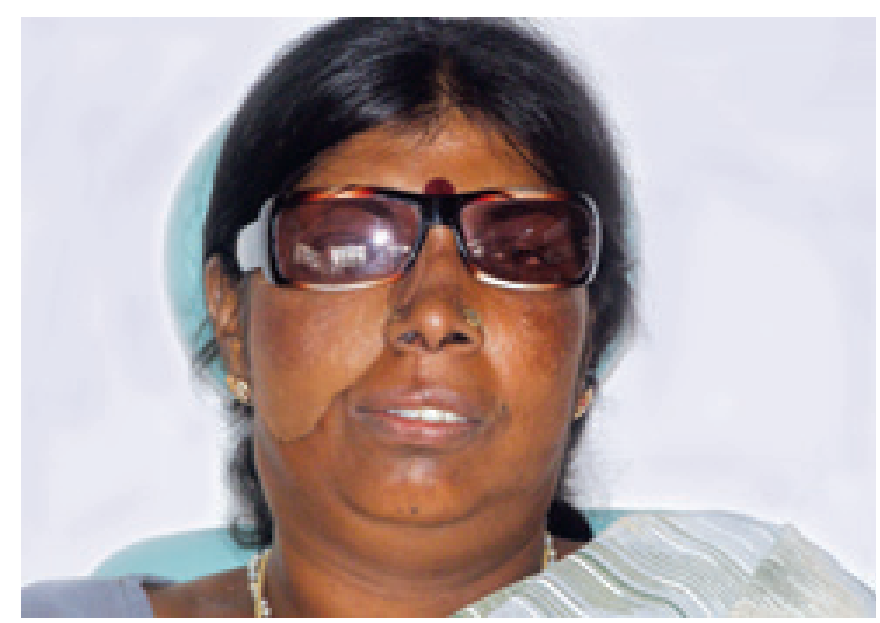

Fig. 7: Definitive cheek prosthesis with spectacles

Fig. 5: Wax patterns with magnets 
the defect in one piece. ${ }^{8}$ A second acrylic tray which fitted exactly onto the acrylic extension of the cast partial framework was designed. Impression material was loaded on the second tray which was fitted onto the acrylic extension and functional moulding of the defect is done. Impression was then removed in two sections and adapted properly outside with the help of indexing holes and projections.

The success of rehabilitative treatment in a patient with extensive resections involving the hard palate, soft palate, and orbital exenteration is directly related to the quality of the prosthesis. ${ }^{9}$ One of the significant problems encountered when fabricating these prostheses is the lack of retention. Retentive elements, apart from conventional adhesives, are normally required. ${ }^{10}$ The use of magnets in multiple/sectional maxillofacial prostheses is an excellent means to bond them together. ${ }^{11}$ However, to assure proper retention and effective stability of the prosthesis, each section should be sufficiently extended and the magnets positioned properly. Additional retention was gained through a pair of spectacles.

The management of a patient with cancer does not conclude with the elimination of the disease but continues with the rehabilitation of function, restoration of esthetics and prevention of infection and maintenance of proper oral hygiene. ${ }^{12}$

\section{SUMMARY}

This clinical report described the prosthetic rehabilitation of a facial defect following right maxillectomy and orbital exenteration. Three-piece prosthesis was designed for the patient, first an obturator to restore the esthetics, phonetics and function, second an orbital prosthesis and third a cheek prosthesis to rehabilitate defective eyes, orbit and cheek prominence. Obturator achieves retention from the remaining teeth while cheek prosthesis is retained by a pair of spectacles and finally the orbital prosthesis is stabilized by magnetic attachments from the above prosthesis.

\section{REFERENCES}

1. Taylor TD. Clinical maxillofacial prosthetics. Chicago: Quintessence Publishing Co Ltd; 2000:233-65.

2. Marunick M, Harrison R, Beumer J. Prosthetic rehabilitation of midfacial defects. J Prosthet Dent 1985;54:553-60.

3. Bulbulian AH. Maxillofacial prosthetics: Evolution and practical application in patient rehabilitation. J Prosthet Dent 1965;15:554-69.

4. Beumer J, Curtis TA, Marunick MT. Maxillofacial rehabilitation: Prosthodontic and surgical considerations. St Louis: Ishiyaku EuroAmerica, Inc 1996:377-453.

5. Srithavaj T, Chotprasert BN, Anun W, Hovichitr W, Sanohkann S. Restoration of large facial prosthesis with a connection to the intraoral prosthesis: A case report. Mahidol Dent J 26:125-29.

6. Keyf F. Obturator prostheses for hemimaxillectomy patients. Journal of Oral Rehabilitation 28;821-29.

7. Aramany, MA. Basic principles of obturator design for partially edentulous patients (Part II): Design principles. J Prosthet Dent 2001;86:562-68.

8. Wiese I, Penkner K, Arnetzl G, Mayer W, Bratschko R. Minimizing movement of an orbital prosthesis retained by obturator prosthesis. J Prosthet Dent 2004;91:188-90.

9. Goiato MC, Fernandes AÚR, dos Santos DM, Barão VAR. Positioning magnets on a multiple/sectional maxillofacial prosthesis. J Contemp Dent Pract November 2007;(8)7:101-07.

10. Guttal SS, Patil NP, Shetye AD. Prosthetic rehabilitation of a midfacial defect resulting from lethal midline granuloma: A clinical report. Journal of Oral Rehabilitation 2006;33:863-67.

11. Brignoni R, Dominici JT. Na intraoral-extraoral combination prosthesis using an intermediate framework and magnets: A clinical report. J Prosthet Dent 2001;85:7-1.

12. Guttal KS, Naikmasur VG, Rao CB, Nadiger RK, Guttal SS. Orofacial rehabilitation of patients with postcancer treatment: An overview and report of three cases. Indian journal of cancer 2010;47:59-64. 